\title{
Abolition de la contrainte par corps et évolution du capitalisme au XIX ${ }^{e}$ siècle
}

Nadine Levratto

\section{(2) OpenEdition}

Journals

Édition électronique

URL : http://journals.openedition.org/ei/341

DOI : 10.4000/ei.341

ISSN : 2553-1891

\section{Éditeur}

Association Économie et Institutions

\section{Édition imprimée}

Date de publication : 30 octobre 2007

Pagination : 221-249

ISSN : 1775-2329

Référence électronique

Nadine Levratto, «Abolition de la contrainte par corps et évolution du capitalisme au XIXe siècle », Économie et institutions [En ligne], 10-11 | 2007, mis en ligne le 31 janvier 2013, consulté le 01 mai 2019. URL : http://journals.openedition.org/ei/341 ; DOI : 10.4000/ei.341 


\section{Abolition de la contrainte par corps et évolution du capitalisme au 19ème siècle}

\section{Nadine Levratto ${ }^{1}$}

Le 19ème siècle a souvent été décrit comme une période de transition entre un capitalisme de gage et un capitalisme de "futurité " au sens de Commons (Stanziani et Levratto, 2008). Dans ce processus d'évolution, la transformation des institutions et des normes se présente comme un facteur clef que les différents courants qui mêlent économie, histoire et droit s'attachent désormais à expliciter. Sans adhérer à une périodisation du capitalisme marquée par des ruptures dont la robustesse a été mise en doute par des recherches d'historiens (Lamoreaux et Rosenthal, 2005 ou Hoffman et al., 2001), nous nous situons ici dans la perspective d'une succession de différentes formes de capitalismes caractérisées par des ordres juridiques particuliers. Les innovations juridiques permettraient ainsi de suivre les changements qui affectent la sphère économique.

La seconde moitié du 19ème siècle est riche en événements de la sorte. Une chronologie frappante se dégage de l'examen des textes de loi adoptés : libération de la création des sociétés, passage d'un droit des faillites exclusivement punitif à un droit qui autorise le "fresh start " (Levratto, 2008), abolition du monopole des courtiers et des agents de change, légalisation du nantissement du fonds de commerce (Stanziani, 2007) et du warrant agricole (Baubeau, 2007), autorisation de l'ouverture des marchés à terme... Ces nouveautés réglementaires favorisent l'ouverture et modernisation des marchés financiers, le développement des banques et du crédit (Gille, 1965 ; Lescure \& Plessis, 1999) et illustrent les mutations radicales de l'économie française au cours de cette période. Parmi les changements institutionnels en relation étroite avec le fonctionnement de l'économie qui se produisent alors, c'est à l'abolition de la contrainte par corps en matière commerciale ${ }^{2}$ que

1 EconomiX-CNRS-Université de Paris 10 Nanterre et Euromed Marseille-Ecole de Management, Adresse : EconomiX -Université de Paris 10 Nanterre - Bâtiment K, 200, avenue de la République, 92001 Nanterre Cedex, E-mail nadine.levratto@u-paris10.fr.

${ }^{2}$ Cette disposition perdure aujourd'hui encore dans le droit civil. La loi du 9 mars 2004 portant adaptation de la justice aux évolutions de la criminalité a remplacé l'ancienne contrainte par corps par la contrainte judiciaire dont le champ d'application est plus étroit. En effet, "En cas d'inexécution volontaire d'une ou plusieurs condamnations à une peine d'amende prononcées en matière criminelle ou en matière correctionnelle pour un délit puni d'une peine d'emprisonnement, y compris en cas 
nous nous intéressons dans ce texte. Le choix de cet événement nous paraît en effet caractéristique de l'expansion du système de crédit qui se produit à cette époque et du passage du capitalisme patrimonial assis sur l'accumulation préalable d'un patrimoine à un capitalisme financier dans lequel la créance se rapproche de plus en plus d'une promesse qui engage essentiellement l'émetteur sur le futur.

Dans ce travail, nous posons l'hypothèse déjà envisagée par des historiens (voir par exemple Vause, 2007) que cette institution est révélatrice du rapport au crédit entretenu par une société ${ }^{3}$. Par extension de cette hypothèse à la relation entre les sphères réelle et financière, cette étude permet aussi de montrer comment le rapport au non remboursement a évolué de manière à favoriser l'esprit d'entreprise et l'activité économique. Actuellement protecteur, le droit de l'entrepreneur en difficulté n'honorant pas ses dettes était à l'origine un droit coercitif dans lequel l'assujettissement physique était utilisé comme moyen de contrainte pour forcer le débiteur à rembourser ses créanciers même si la loi prévoyait elle-même les limites de son application ${ }^{4}$. A travers ce texte nous nous intéressons aux changements qui ont touché les dispositifs juridiques et à la manière dont les modifications apportées ont accompagné l'évolution du processus d'accumulation. Deux thèses juridiques seront conjointement mobilisées. La première, qui est aussi la plus connue des économistes du droit ${ }^{5}$ se réfère à l'autorité des textes dont les

d'inexécution volontaire de condamnations à des amendes fiscales ou douanières, le Juge de l'application des peines peut ordonner, dans les conditions prévues par le présent titre, une contrainte judiciaire consistant en un emprisonnement dont la durée est fixée par ce magistrat dans la limite d'un maximum fixé par la loi en fonction du montant de l'amende ou de leur montant cumulé. " (Article 749 du Code de procédure pénale, dans sa rédaction issue de la Loi du 9 mars 2004).

3 A titre d'illustration de cette thèse, on citera ce long extrait de Moreau-Christophe (1841) " on ne peut nier que, quelle que soit l'origine des dettes, elles n'accusent un véritable état de gêne de la part de celui qui ne peut les payer. Lors donc qu'un grand nombre d'effets de commerce sont protestés faute de paiement, lorsque des faillites se déclarent et que des bilans sont déposés, lorsque des saisies sont pratiquées sur les meubles, sur les immeubles ou sur la personne des débiteurs, lorsque des poursuites sont exercées et des condamnations prononcées contre eux, lorsque enfin la contrainte par corps s'empare du débiteur lui-même et le place en séquestre sous les verrous, alors ces faits, s'ils sont nombreux, graves, persistants, témoignent hautement de la misère du pays ou des individus qu'ils concernent. C'est pourquoi nous plaçons les dettes au nombre des signes indicateurs de la misère. "

4 D'après l'ordonnance d'avril 1673, la contrainte par corps ne devait intervenir que dans des cas bien déterminés: pour obtenir la représentation de meubles saisis, l'exécution de contrats maritimes, le paiement de fermages, le paiement de dommagesintérêts, etc. En raison de leur grand âge, les septuagénaires bénéficiaient d'une dispense (art. 9, tit. 34). Plus tard, ce furent les femmes, les mineurs et les petits débiteurs qui furent épargnés (Ferrière (1755) t. 1, pp. 548-551 et Merlin de Douai (1812-1826) t. 3, pp. 64 et ss.).

5 C'est celle qui fonde les travaux de la Law and Economics qui propose une lecture des dispositifs juridiques dans laquelle les textes occupent la première place même si l'observation de pratiques situées et la narration de cas viennent l'enrichir. 
changements constituent la cause première de l'évolution des structures et des comportements économiques. La seconde approche de la relation entre la norme juridique et le monde de l'économie passe par un "juge-entraineur " (Ost, 1983) qui construit l'avenir en participant à une vaste négociation entre l'ensemble des partenaires intéressés par le devenir d'une entreprise. Des travaux préalables ont montré qu'elle était particulièrement adaptée à l'analyse de l'évolution du droit des faillites (Hautcoeur \& Levratto, 2007). Nous verrons ici que les pratiques des tribunaux ont souvent auguré des modifications de la loi et que si la contrainte par corps a fait partie $\mathrm{du}$ dispositif législatif français en vigueur jusqu'en 1867, son application par les tribunaux était des plus réduites.

La suite de ce travail sera organisée de la façon suivante. A partir d'une présentation de la chronologie de l'abolition de la contrainte par corps et la mise en relation des pratiques observées et des modifications de la loi, la première partie montre comment la contrainte par corps bridait l'esprit d'entreprise et, pour cette raison, ne pouvait demeurer en place. La fin de la prison pour dettes concernant les commerçants n'est cependant qu'une condition permissive de la liberté d'initiative économique caractéristique du 19 ème siècle. La deuxième partie traite ainsi des changements qui ont affecté le droit des faillites dans le sens d'une atténuation des conséquences de la prise de risque pour les commerçants. Mais le contexte institutionnel a également répondu au besoin d'accumulation de l'époque en créant les outils qui permettent de s'affranchir du poids du passé. La troisième partie rend compte des dispositifs juridiques qui ont ouvert la voie à un mode de production dans lequel les promesses, les anticipations et l'immatériels deviennent des gages reconnus dans le processus d'attribution des crédits. Aucun de ces changements n'aurait pu se produire sous un régime de financement soumis à la contrainte par corps.

\section{De la mise en place de la contrainte par corps à son abolition : bref récit d'un long parcours}

La procédure collective, protection recherchée par les débiteurs en difficulté, semble ne pas prévoir véritablement de contraintes à l'égard des personnes en faisant l'objet. Pourtant, la législation relative aux entreprises en difficulté et la tolérance à l'égard des débiteurs et des faillis sont construites sur le Code de commerce de 1807, lui-même élaboré sur la base de l'ordonnance de 1673, dont la sévérité a été maintes fois soulignée. La contrainte par corps exercé sur le débiteur à la demande des créanciers en 
constitue l'une des dispositions les plus controversées. Cela vaut dès son origine ${ }^{6}$.

\subsection{Origine et justification de la contrainte par corps}

A l'œuvre dans le droit romain 7 , également présente sous une forme encore plus marquée dans la loi salique à l'origine du droit germanique $^{8}$ et diffusée dans la plupart des droits nationaux, la contrainte par corps est la manifestation la plus ancienne du droit des obligations. La barbarie et/ou l'inefficacité économique de la mise en esclavage des débiteurs en vigueur dans l'Empire romain seraient à l'origine d'un édit pris en 118 av. J C. par lequel est instaurée une action sur les biens du débiteur; il s'agit de la venditio bonorum (ou vente des biens). Elle se présente comme l'une des premières dissociations entre la personne du débiteur et la dette. En cas de non remboursement, le prêteur va tout d'abord prendre une mesure conservatoire sur le patrimoine du débiteur. A partir de ce moment, celui-ci est gelé ${ }^{9}$ et placé sous la surveillance d'un curateur qui en organise la vente. L'acquéreur des biens se substitue au failli dont il doit rembourser les dettes. Même si la saisie des biens remplace l'emprisonnement et, parfois, l'exécution du failli, la contrainte par corps n'en demeure pas moins pratiquée, que le débiteur soit commerçant ou non et que son incapacité de paiement soit volontaire ou fortuite. Car, si le morcellement du droit auquel procède le Moyen-âge ${ }^{10}$ touche surtout le domaine d'application et l'organisation des procédures ${ }^{11}$, dans cet ensemble apparemment

\footnotetext{
${ }^{6}$ L'évolution historique des articles 1265 à 1270 du Code civil français, abrogés par la loi du 9 juillet 1991, qui réglementaient la cession de biens définie comme " l'abandon de tous les biens du débiteur à ses créanciers et ce dès l'instant où il se trouve dans l'impossibilité de payer ses dettes " est analysée par Zambrana-Moral (2004).

7 Du 5ème au 2ème siècle avant J.C. à Rome, la loi des XII Tables livrait le débiteur à merci de son créancier : si des transactions étaient possibles, en fait les débiteurs (nexi) étaient enchaînés, en butte aux pires traitements, passibles de mort, de vente, sans protection légale effective (Desurvire, 1991). Elle fut remplacée par la loi Poetelia Papiria (326 av. J.-C.) qui, quoi que moins cruelle, ne mit pas fin à une pratique consistant à enchaîner les voleurs attribués par le préteur à la victime du délit (addicti) ; elle maintenait en outre l'exécution sur la personne des débiteurs judicati et addicti, c'est-à-dire jugés et attribués par le magistrat à leur créancier. Pour une présentation détaillée et une discussion sur le caractère plus ou moins systématique des traitements violents infligés aux endettés, voir Kalnin-Maggiori, 2004.

8 En cas d'inexécution de la fides facta (foi jurée germanique en vigueur dans les contrats formels), le débiteur récalcitrant est frappé d'amende, puis sommé trois fois par le créancier qui doit l'attendre jusqu'au coucher du soleil. Il peut ensuite saisir ses biens et le retenir prisonnier (voir Brissaud, 1908, pp. 427-431).

${ }_{9}$ Il ne pourra plus créer des dettes nouvelles ni vendre des biens lui appartenant.

${ }_{10}$ L'ancien droit distingue trois droits de la faillite : le droit du midi, le droit des foires et celui de la Coutume de Paris. Pour une étude de la contrainte par corps au Moyenâge, voir J. Claustres, 2007.

11 Le midi de la France connaît encore à cette époque la venditio bonorum, les provinces régies par la Coutume de Paris connaissent une organisation collective avec
} 
hétéroclite on peut faire ressortir deux traits communs que sont le paiement des créanciers comme objectif primordial et une répression accentuée à l'égard du débiteur, qui soit ou non de bonne foi. Ces usages furent abrogés par une ordonnance royale de 1629 pour être remplacés par la prison pour dettes.

Cette pratique sera reprise dans l'ordonnance royale de 1673 qui unifie le droit commercial en France. Connu sous le nom d'ordonnance Colbert ou de Code Savary (du nom de son rédacteur), ce texte marque une grande avancée en matière de difficulté des entreprises en cherchant à établir des distinctions à l'origine d'une gradation dans la gravité des comportements du débiteur défaillant. La principale est celle entre le failli simple et le banqueroutier. Le failli simple est celui que l'on appellerait à l'heure actuelle le débiteur de bonne foi ou malchanceux "victime d'accidents imprévus"12. Il bénéficie d'une certaine clémence, surtout au regard des peines qui s'appliquent au banqueroutier frauduleux dont les malversations ont provoqué la ruine. Tous deux sont sanctionnés, mais de manières différentes. Le failli simple était par sa seule déconfiture considéré comme un individu dangereux (Chaput, 1996) voire nocif pour la bonne marche de l'économie. C'est pourquoi, dès l'ouverture de la procédure de faillite, à coté des déchéances diverses dont il était frappé ${ }^{13}$, il pouvait faire l'objet de la contrainte par corps à la demande des créanciers. La banqueroute frauduleuse, quant à elle, s'accompagnait systématiquement d'une peine de prison: pour dettes ou pour la commission d'actes frauduleux tels que définis dans deux articles de l'ordonnance ${ }^{14}$.

L'usage de la contrainte par corps pour les commerçants doit également être compris par rapport au risque spécifique que présente

contribution des créanciers au "sol la livre", le droit des foire connaissait une procédure de saisie des biens du débiteurs dans son pays d'origine (voir Szramkiewicz, 1989, p.182).

12 La faillite est définie par Miromesnil en 1778 comme étant la nécessité ou se trouve un débiteur de bonne foi ou par des accidents imprévus ou par des malheurs, de demander à ses créancier terme et délais pour payer ses engagements ou une partie de ceux-ci.

13 Il s'agit principalement de l'exclusion du monde du commerce, de l'interdiction de réaliser toute opération de change et de la défense d'entrer à la bourse et déchéance des fonctions publiques. Ces déchéances répondent plus à des objectifs de sûreté, de protection des tiers qui font que le droit des faillites est aussi une législation d'ordre public qu'à un souci de réprobation publique du failli (Schwartz, 1998, p. 1808

14 L'article 10 condamne le "divertissement d'effet" qui correspond à une dissimulation d'éléments d'actif avant ou après l'ouverture de la procédure de faillite. L'article 11 concerne le non-respect de ses obligations comptables par le débiteur et tout particulièrement la production d'un bilan " gonflé ". Le débiteur peut simuler une hausse de l'actif dans le dessein de dissuader les créanciers de faire valoir leurs droits. Ceux ci risquant d'engager des frais élevés pour n'obtenir qu'une infime partie de leur créance impayée préféreront abandonner les poursuites. La hausse simulée de l'actif est une technique permettant au débiteur d'obtenir un concordat de la part de ses créanciers croyant son insolvabilité passagère et non définitive. 
le paiement à distance (distancia loci) et qui se traduit par l'usage de la lettre de change. Celle-ci exposait toujours à l'emprisonnement puisqu'elle n'était pas assortie de collatéraux réels. En revanche, les non commerçants, la plupart du temps détenteur d'un capital tangible servant de caution au prêt, échappaient à la menace sur la personne. Des abus liés à l'usage de lettres de changes fictives permettant de transformer les débiteurs en quasi-commerçants sont recensés au cours de la première moitié du 19ème siècle par et par Balzac $^{15}$. La menace de la contrainte par corps est donc brandie par les créanciers pour "forcer le paiement des pauvres, les forcer à vendre leurs biens à la sauvette, les brader, en contradiction avec les lois qui imposent des délais qui sont des protections" (BayleMouillard (1836) p. 230). La contrainte par corps est donc moins un instrument de sécurisation de la dette qu'un complément des pratiques usuraires dans lesquelles "le créancier compte moins sur la capacité de remboursement du débiteur que sur la honte de ses proches pour obtenir le remboursement de ses prêts " (Hautcoeur, 2008, p. 132).

\subsection{Des premières suspensions à l'abolition définitive}

De multiples demandes en faveur de l'abandon de ce dispositif sont exprimées sous l'ancien régime ${ }^{16}$ et demeureront sans effet pendant plus d'un siècle. L'application de la contrainte par corps est pourtant abondamment dénoncée dans des écrits qui en soulignent le manque d'humanisme (Anonyme, 1860). Il nous semble toutefois que ce sont surtout des arguments d'ordre économique qui vont permettre à ses détracteurs d'obtenir son abolition à la fin du 19ème siècle. Pour l'anecdote, on notera que celle-ci se produit quasi simultanément en France où la loi de 186717 supprime cette pratique et au Royaume-Uni où son usage était encore plus marqué et qui en

\footnotetext{
15 "Postel, dit Madame Chardon en entrant sans voir David, consent à prêter les mille francs, mais pour six mois seulement, et il veut une lettre de change de toi, acceptée par ton beau-frère, car il dit que tu n'offres aucune garantie" (Balzac, 1837, p. 143).

16 Dès les États Généraux de 1560, les marchands du tiers-état demandent un relâchement de la contrainte par corps pour les commerçants. "Les marchands dudit tiers-état supplient ledit seigneur roi avoir pareil privilège pour leurs dettes, qu'ont ceux de Lyon et autres villes qui emportent obligation par corps, et principalement quand ils ont à faire de marchand à marchand pour les réduire à gros intérêts, qu'ils sont contraints de payer ..." (p. 438). "Recueil des Cahiers Généraux des Trois Ordres" (1789) Tome 1, chez Barrois l'aîné, Paris. Cette demande sera précisée dans les cahiers de doléance de 1614 qui demandent que les poursuites concernent seulement les fraudeurs et banqueroutiers. On retrouvera cette réclamation dans les cahiers de 1789 (Hilaire - 1992).

17 La loi du 22 juillet 1867 interdit expressément la contrainte par corps pour obtenir l'exécution forcée du débiteur en matière civile (la contrainte par corps demeure cependant en droit pénal (elle se nomme désormais contrainte judiciaire), car le paiement de la dette concerne l'intérêt public et non l'intérêt privé d'un créancier).
} 
abandonne toutes les formes en 1869 (voir Markham Lester, 1995). Cette concomitance nous paraît révélatrice des transformations du capitalisme au 19ème siècle. La fin de la contrainte par corps marque en effet une rupture formelle déterminante : le gage le plus fort que le débiteur pouvait donner, à savoir lui-même, est désormais exclu de la transaction. Les changements qui interviendront par la suite ne feront que prolonger la tendance entamée à cette époque.

La première expérience de suppression a lieu en matière civile en mars 1793. Si l'Assemblée constituante avait maintenu la contrainte par corps dans la Loi du 16 Août 1790 (titre 12, article 5) son abolition est exprimée par le décret du 9 mars 1793. Ce changement a lieu en pleine révolution jacobine, alors que les besoins d'hommes de troupes liés aux guerres dans lesquelles est plongée la France sont intenses ${ }^{18}$. "C'était donner un champ libre à la mauvaise foi, fit observer Bigot-Préameneu, dans un temps où le besoin de la comprimer était le plus pressant " (cité par M. Martin, 2003). Peut-être faut-il chercher là les causes de son rétablissement par un décret du 24 ventôse An V dont la mise en application va se révéler laborieuse ainsi que l'attestent les nombreux débats autour de la loi du 15 germinal An VI qui en organise le principe (Dalloz, 1851 , p. 423). Sur les trois titres que compte ce texte, deux - l'un relatif à la contrainte par corps et l'autre à l'emprisonnement seront abrogés par le Code civil et de procédure. Seul restera en place le titre 2 relatif à la contrainte en matière commerciale.

Le Code de Commerce de 1807 constitue une étape décisive dans l'évolution du droit des entreprises en difficulté. La matière répressive de cette législation conserve une consistance certaine (Hautcoeur et Levratto, 2007) tout en s'affinant par le biais de l'établissement de nouvelles distinctions entre les comportements pouvant entraîner la sanction de ceux qui les commettent. Par la distinction qu'il opère entre les débiteurs malchanceux, incompétents et malhonnêtes, le Code de commerce permet la gradation des déchéances et des peines appliquées. Si la contrainte par corps s'applique formellement à tous dès la déclaration de la faillite, elle est mise en œuvre de manière très différenciée selon les juridictions. Le clivage entre Paris et la province y apparait de manière criante car seul Paris offre un nombre de détenus pour dettes suffisamment élevé (voir le tableau ci-dessous) pour permettre un établissement distinct et séparer les détenus pour dettes des

${ }^{18}$ Cette période d'effort de guerre intense est également caractérisée par la gigantesque inflation des assignats qui perturbe l'activité économique et appauvrit la population. Les réquisitions et au "maximum général des prix et des salaires ", procèdent de la volonté d'empêcher les émeutes de la faim qui risqueraient de porter atteinte à l'effort de guerre (Asselain, 1984, p. 119) l'abolition de la contrainte par corps peut être considérée comme le résultat d'une recherche d'apaisement social. 
autres ${ }^{19}$. Dans de nombreux autres départements, il n'est pas rare de ne compter que quelques détenus pour dettes. C'est tout particulièrement le cas dans les ressorts ruraux tels que les Hautes et Basses Alpes, l'Aude, le Cher, etc. qui n'en comptent aucun (voir Lucas, 1836, p. 194).

Tableau 1 : Nombre de détenus pour dettes en $1830^{20}$

\begin{tabular}{|l|l|l|l|l|l|l|l|l|l|l|}
\hline & \multicolumn{2}{|c|}{ France entière } & \multicolumn{2}{c|}{ Seine } & \multicolumn{2}{c|}{$\begin{array}{c}\text { Seine } \\
\text { Inférieure }\end{array}$} & \multicolumn{2}{c|}{$\begin{array}{c}\text { Pas de } \\
\text { Calais }\end{array}$} & \multicolumn{2}{c|}{ Rhône } \\
\hline & Hommes & Femmes & H & F & H & F & H & F & H & F \\
\hline $1^{\text {er Janv. }}$ & 725 & 42 & 223 & 21 & 31 & 2 & 22 & 4 & 30 & 2 \\
\hline $1^{\text {er }}$ Avr. & 712 & 44 & 213 & 14 & 31 & 2 & 23 & 4 & 20 & - \\
\hline $1^{\text {er Juil. }}$ & 744 & 38 & 238 & 10 & 31 & 2 & 22 & 6 & 21 & - \\
\hline $1^{\text {er }}$ Oct. & 501 & 29 & 54 & 5 & 27 & 1 & 19 & 4 & 19 & - \\
\hline
\end{tabular}

(Source : Lucas, 1836)

Des données minutieusement collectées par Bayle-Mouillard entre 1830 et 1832 complètent ce tableau: une détention par an pour 117 habitants dans la Seine, contre une pour 541 dans le Rhône, une pour 73712 dans les Deux-Sèvres et une seule en Vendée sont révélateurs des écarts entre départements étudiés par ce fondateur de la statistique judiciaire (Hautcoeur, 2008)

Consécutive à plusieurs projets allant dans le sens de l'abolition dont deux particulièrement avancés présentés sans succès au parlement en 1828 et 1829 , la loi du 17 avril 1832 donne le " coup d'envoi" de la lente érosion des sanctions frappant le failli. Elle écarte de l'application de la contrainte par corps plusieurs catégories de débiteurs (les femmes et filles non légalement réputées marchandes publiques, les mineurs, les veuves et les héritiers, les débiteurs de plus de 70 ans, etc.) et de créanciers qui peuvent la requérir ${ }^{21}$ (Voir Alauzet, 1857, p. 421 pour un commentaire de ces nouvelles dispositions). Le champ d'application et les conditions de la mise en œuvre de la contrainte par corps sont précisés par la loi du 17 avril 1832 qui fixe à 200 francs minimum la dette contre laquelle on peut y recourir et établit un barème proportionnel pour la durée de l'emprisonnement avec un maximum de 5 ans pour les dettes de

\footnotetext{
19 Traditionnellement détenus à la prison Sainte-Pélagie, les détenus pour dettes parisiens ont été transférés en 1834 à la maison pour dettes de la rue de Clichy construite à cette fin. Le roman du 19ème siècle offre de nombreuses descriptions de ces deux maisons d'arrêt. (Cf Mayret, 1834)

20 On notera à ce propos une différence importante avec le Royaume Uni. Ainsi, au 29 avril 1826 la France comptait 821 détenus pour dettes soit 1 pour 39538 habitants. A la même datte, on en recensait 3820 en Angleterre, ce qui donne un rapport de $1 / 6$ 639. C'est de cette époque que date la préparation du premier Bill pour l'abolition de la prison pour dettes en Angleterre. Voir Lucas, 1836, p. 197.

${ }^{21}$ Les demandes émanant de membres de la famille du débiteur (épouse, enfants, parents, même éloignés) ne sont plus recevables.
} 
5000 francs et plus. Le gouvernement provisoire la supprimera en 1848 mais elle sera rétablie par l'assemblée constituante à la fin de la même année. Pourtant, la loi du 13 décembre 1848 marque une accélération dans le processus d'abolition définitive de la contrainte par corps en réduisant de manière générale toutes les durées d'incarcération qui vont de 3 mois pour les dettes de 50 francs à 3 ans pour celles de 6000 francs et davantage ${ }^{22}$.

Les lois du 17 avril 1832 et du 13 décembre 1848, "se mettant au dessus des fausses théories sentimentales et philanthropiques qu'on mettait en avant pour faire décréter l'abolition de la contrainte par corps en matière commerciale, ont su la conserver au crédit, en y apportant les adoucissements et les améliorations indiquées par l'expérience et commandées par l'humanité " (Dalloz, 1851, p. 424). Selon ses détracteurs, le statut juridique de la contrainte par corps et son champ d'application effectif contribuent à la rendre peu efficace donc superflue. En premier lieu, on rappellera qu'avec la contrainte par corps, ce n'est pas à une peine mais à une mesure visant à protéger les intérêts du créancier que l'on a affaire. Elle vise en tout et pour tout à empêcher la disparition du débiteur. Dans le cadre de son incarcération, le failli n'est pas placé sous la surveillance de l'autorité publique; c'est au directeur de la prison à répondre de la représentation du prisonnier. "... le contrat se ferme alors entre le directeur et la créancier incarcérateur, aussi la première obligation de celui-ci est-elle de consigner par avance les aliments pour le débiteur incarcéré qui doit être nourri à ses frais tant qu'il est retenu prisonnier " (Teulet, 1835, p. 362). Bayle-Mouillart (1836) évalue le coût total pour le créancier à environ 900 francs. Même si la contribution alimentaire fera très rarement l'objet d'une revalorisation au cours du 19ème siècle, cette prise en charge des incarcérés par leurs créanciers va contribuer à nourrir l'image du débiteur vivant bien dans sa prison. En second lieu, les critiques de la contrainte par corps rendent compte de l'absence presque totale de commerçants dans les prisons pour dettes. La contradiction entre un dispositif sensé garantir la bonne marche du commerce et la structure de la population des détenus fait qu'au bout du compte la contrainte par corps profite peu à ceux pour lesquels elle a été instaurée. Le faible nombre de personnes restant incarcérées et la modestie des sommes en jeu renforcent l'argumentaire. Car, de fait, les montants en cause sont souvent très faibles même s'ils varient selon les sources. Ainsi, sur 3000 contraintes qui se décernent annuellement à Paris, on compte moins

22 Ces indications s'appliquent aux français. Les durées d'incarcération resteront longtemps plus importantes pour les étrangers en France et pour les indigènes dans les colonies. 
de 700 incarcérations ${ }^{23}$ qui pour les deux cinquièmes concernent des sommes de 200 à 1000 francs (Anonyme, 1860, p. 21). Selon Lucas (1836) sur 4246 détenus pour dettes à Paris sur une période de 10 ans, 125 représentaient une dette supérieure à 10000 francs.

La loi du 22 juillet 1867 abolit enfin la contrainte par corps en matière civile et commerciale pour ne la maintenir qu'en matière criminelle, correctionnelle et de simple police à raison des condamnations à l'amende, aux frais, aux restitutions et aux dommages-intérêts ${ }^{24}$. Le 25 juillet, les 60 prisonniers pour dettes encore enfermés à la prison de la rue de Clichy sont libérés. Ce texte marque une date clef dans le processus de mise en place d'une grande liberté du commerce. Il est également l'aboutissement des débats autour du bien-fondé de la contrainte par corps comme instrument permettant de moraliser la vie des affaires et garantir la bonne marche de l'économie. Malgré la précocité des dénonciations de cette pratique jugée à la fois inhumaine et inefficace, l'abolition de la contrainte par corps s'est opérée lentement. On observe la même répugnance à en expurger le droit au Royaume-Uni où elle disparaît en 1869 et aux Etats-Unis où elle est maintenue en cas de faillite frauduleuse jusqu'en 1841. Si l'abolition de ce dispositif peut paraitre anecdotique compte tenu du petit nombre de cas concernés et la faiblesse des montants en jeu, les changements intervenus nous paraissent révélateurs des mutations du capitalisme de l'époque. C'est pourquoi nous allons maintenant nous attacher à mettre en relation la fin de la contrainte par corps et d'autres dispositifs juridiques qui attestent également des changements du capitalisme au cours du 19 ème siècle.

\section{Le droit des faillites comme instrument de protection des commerçants}

Systématiquement décrié par les juristes, les débiteurs, les banquiers et les chambres de commerce comme l'attestent les critiques adressées au Livre III du Code de commerce dès sa promulgation, le droit des faillites, à l'origine réservé aux commerçants, n'en exerce pas moins un certain attrait pour les

\footnotetext{
${ }^{23}$ Paris est réputé représenter le tiers des incarcérations alors qu'il représente moins d'un tiers de l'activité économique du pays. Les villes industrielles de province recourent moins à la contrainte par corps. Au 1 $1^{\text {er }}$ janvier 1831 Marseille comptait ainsi 3 détenus pour dettes

24 Toutefois, par l'application de l'art. $455 \mathrm{du}$ code de commerce, la contrainte par corps ne peut, même pour ces causes, être exercée contre un failli ou une personne en état de liquidation judiciaire (Lyon-Caen et Renault, Précis de Droit Commercial, $\mathrm{n}^{\circ}$ 2688, cité dans Guyot \& Raffalovitch (1901), Tome 1, à l'article "Contrainte par corps"). Cette disposition ne s'appliquera vraiment qu'en France métropolitaine. Ainsi, également supprimée dans les colonies, elle fut rétablie dans les comptoirs français de 1'Inde à l'égard des indigènes par un décret du 12 février 1898.
} 
différentes catégories de population, au point que son domaine d'application a fait l'objet d'un élargissement jusqu'aux personnes physiques. Dès le 19ème siècle, les manuels de droit commercial insistent sur les précisions à apporter à la définition et à l'identification des droits et devoirs auxquels doivent se conformer " ceux qui exercent des actes de commerce et en font leur profession habituelle " (Code de commerce, Livre I ; Titre 1). Dans cet ensemble c'est à la détermination des bénéficiaires du statut de commerçant donc du droit des faillites - et au fonctionnement des tribunaux de commerce où se jugent les litiges entre débiteurs et créanciers qui nous nous intéressons tout particulièrement.

\subsection{Qui est commerçant?}

Le terme "commerçant" a été introduit par le code de commerce de 1807. Auparavant, on parlait de marchands, de négociants, de banquiers et d'artisans. A la fin de l'Ancien Régime, les juridictions consulaires étaient compétentes ratione personae pour les litiges entre commerçants, mais la loi ne définissait pas ce terme et la seule appartenance à une corporation a été jugée insuffisante. Les nobles, qui étaient interdits de commerce sous peine de dérogeance, contournaient cette règle en devenant commanditaires, et certains se sont ainsi immiscés dans la gestion des sociétés sans pour autant accéder au statut de commerçant. A partir du Code civil, le champ d'application du statut de commerçant ne va cesser de s'élargir, traduisant la volonté du monde des affaires de bénéficier du traitement prévu par le Code de Commerce, notamment en matière de faillite. C'est d'ailleurs cet agrandissement du périmètre d'application de la loi qui conduit certains auteurs (Marco, 1992 et Di Martino, 2005) à expliquer l'augmentation régulière du nombre de faillites au cours du 19ème siècle.

La question que se pose est celle des causes qui conduisent les débiteurs à préférer l'application du régime des faillites à celui des déconfitures ${ }^{25}$. Est-ce en raison de la plus grande capacité à recommencer une activité de commerçant qu'autorise le premier depuis la " décodification " de 1838, qui s'est révélée de plus en plus favorable à la survie des entreprises et au maintien des entrepreneurs dans le monde économique ? Plaide en faveur de cette interprétation la mise en place du contrat par abandon d'actif introduit par la loi du 17 juillet 1856 qui décharge le failli de

\footnotetext{
${ }^{25}$ L'extension du régime de la faillite à des secteurs qui n'en relevaient pas (mines par exemple) est souvent interprétée comme le produit de la demande des justiciables qui cherchent à se placer sous la protection du texte le plus avantageux de leur point de vue (Deffains, 2007). "Il n'est pas rare de voir un débiteur qui a plaidé pour prouver qu'il n'est commerçant afin d'échapper à la contrainte par corps, plaider ensuite qu'il est dans le commerce pour faire admettre sa faillite" (Bayle-Mouillard, 1836, p. 215).
} 
l'administration de ses biens, lui permet de rentrer après son concordat dans la vie commerciale et cesse de l'exposer aux poursuites des créanciers. A contrario, le régime de la banqueroute, toujours défavorable aux commerçants, tombera peu à peu en désuétude. En effet, le banqueroutier simple ne peut plus demander à bénéficier de la liquidation et le banqueroutier frauduleux ne peut plus prétendre ni au concordat, ni à l'excusabilité ou à la réhabilitation (Guyot et Raffalovitch, 1901, article "Faillite"). Concernant les cessations de paiements des non commerçants, la déconfiture ne protège l'affaire d'aucune façon: la procédure collective n'est pas appliquée, d'autant qu'aucune partie organisée ne la demande alors qu'elle pourrait être très avantageuse : pour les paysans, la crainte de l'expropriation conduit à les écarter durablement du champ d'application du droit des faillites.

Les avantages que les commerçants tirent d'un droit fait pour eux et mis en pratique par eux expliquent en grande partie l'abondante jurisprudence relative à l'article 437-1 consacré à la définition de la qualité de commerçant qui ouvre le livre III "des faillites et banqueroutes ". Pas moins de 101 commentaires sont relevés par Dalloz et Vergé (1877, pp. 547-549) ; un même travail de recensement de la doctrine dans ce domaine est réalisé par Tripier (1902, pp. 640-641), prouvant ainsi l'intensité des débats sur l'interprétation du statut de commerçant et sur les possibilités d'y accéder. L'évolution du droit de la faillite observée en France témoigne de l'unification du droit commercial et du droit civil qui a admis la faillite des personnes morales de droit privé non commerçantes (sociétés civiles, associations, syndicats, coopératives), a autorisé le redressement judiciaire des artisans en 1985, celui des agriculteurs depuis 1988 et a permis l'extension de la liquidation des sociétés à leurs dirigeants qui ne sont pas toujours juridiquement des commerçants. Ce même phénomène de rapprochement s'est produit néanmoins avec une temporalité sensiblement différente dans d'autres pays d'Europe : en Angleterre, l'Insolvency Act (1986) s'applique à tous les débiteurs, en Allemagne, la procédure du concours (1877) est également commune à tous les débiteurs insolvables (ce qui explique le maintien de ce régime particulier dans les trois départements recouvrés d'Alsace-Lorraine), de même qu'aux Pays-Bas (voir Sgard, 2005).

Le régime de la faillite et la protection qu'il offre sont ainsi prisés par les débiteurs qui, en vertu du principe de la masse, ne risquent pas de se trouver confronté à des créanciers isolés cherchant à engager les poursuites les premiers de crainte de se trouver dépassés par d'autres. 


\subsection{Des juges de commerce aux attributions essentielles}

Juridiction particulière au sein de l'organisation judiciaire, les tribunaux de commerce font systématiquement l'objet de critiques dans leur fonctionnement. Depuis leur institution, leurs prérogatives dans la procédure de faillite ont pourtant été confirmées. Arbitres du moment où l'entreprise passe de l'univers de l'économie marchande où dominent les contrats et la propriété privée à celui du contentieux qui organise l'expropriation légale des propriétaires, les juges se révèlent ainsi des éléments clés de la faillite comme institution du capitalisme.

La faillite n'étant en rien un état naturel, la question de la définition de la date à laquelle intervient la cessation de paiement a très tôt occupé les tribunaux et les auteurs de manuels et de guides à l'usage des praticiens. Dans le tome $1 \mathrm{du}$ "Dictionnaire des faillites ", Mascret recense les différentes conceptions de l'état de cessation de paiement et rappelle le point de vue de P-S. Boulier-Paty exprimé dans l'ouvrage "Des faillites et banqueroutes " pour qui " il s'agit moins ... pour le commerçant d'être solvable ou insolvable, que de savoir, si, de fait, il paye ou ne paye pas : quel que soit son actif, fût-il supérieur à son passif, s'il cesse de payer, il est en état de faillite. Au contraire, si, par un crédit toujours soutenu, il fait constamment honneur à ses engagements, dût-il plus qu'il ne possède, il n'est point en faillite" (Mascret, 1863, p. XXIII). Le commentaire analytique de la loi du 8 juin 1838 effectué par F. Lainné en 1839 semble dissocier la situation comptable de l'affaire de la situation de faillite, l'auteur considérant que "...c'est aux juges qu'il appartient de décider, d'après les circonstances, si la suspension de payements équivaut à une cessation réelle... " (Lainné, 1839).

Au-delà du contrôle qu'ils exercent sur les modalités de l'application du droit, les juges de commerce possèdent ainsi et surtout le pouvoir de discriminer entre une situation d'illiquidité temporaire et une situation d'insolvabilité, cette dernière étant un préalable nécessaire mais non suffisant de la faillite. Cette disposition apparaît précocement car, si les textes de droits archaïques prévoyaient, dans un cadre civil, essentiellement des procédures d'exécution qui pouvaient varier selon les suzerainetés locales, le Titre IX de l'ordonnance de commerce de 1673 précisait que "La faillite ou banqueroute sera réputée ouverte du jour que le débiteur se sera retiré ou que le scellé aura été apposé sur ces biens " (Bravard-Veyrières, 1840, p. 617). Cette "...volonté du législateur royal, d'imprimer une clarté juridique dans le déclanchement de la procédure "(Desurvire, 1992, p. 39) consacre l'absence de critère absolu de définition de la faillite et, en conséquence, l'importance de l'exercice de jugement opéré par les 
tribunaux. Les manuels de droit commercial sont clairs à ce sujet : les tribunaux ont pour mission de déclarer la cessation de paiement, ils sont également souverains pour apprécier les circonstances et les faits qui relèvent de la cessation de paiement, ce qui les conduit en particulier à arrêter la date de la cessation de paiement. Cette disposition est essentielle car le jour d'ouverture de la faillite permet de fixer la "période suspecte ", c'est-à-dire la période précédant l'ouverture de la faillite pendant laquelle le débiteur peut avoir passé des actes plus ou moins frauduleux, dont les créanciers peuvent demander l'annulation.

Le rôle clef joué par les juges dans l'acte de faillite éloigne définitivement cette procédure de l'image de lutte des faibles (les débiteurs) contre les forts (les créanciers) à laquelle la loi du marché est souvent associée. La rupture du contrat par l'entrepreneur ne le met pas pour autant hors jeu. Au contraire, il sort d'un système institué, le marché monétisé, régulé par la discipline des contrats et des droits de propriété, pour entrer dans un mécanisme juridictionnel de gouvernance et de distribution du revenu. Ce type d'avantage expliquera les efforts de nombre de catégories de personnes impliquées dans la vie des affaires pour bénéficier de l'application du droit des faillites.

\section{L'abolition de la contrainte par corps, condition permissive d'un capitalisme fondé sur la dette bancaire}

L'histoire économique enseigne que le 19ème siècle marque la transition entre une économie dominée par la propriété foncière et un système dans lequel la finance joue un rôle essentiel. Cette transition est souvent liée à la liberté contractuelle qui domine l'époque (Atiyah, 1979). Nous ne reprenons pas ici la thèse, d'un 19ème siècle caractérisé par le passage d'une norme légale de droit divin immuable à une Common Law dominée par le contrat et dont l'application est déterminée par le contexte institutionnel et social (pour une critique générale de cette vision de la rupture voir Polanyi, 1983 ; des considérations plus techniques sur le droit sont plutôt mobilisées par Farrar, 1981). Fidèles à la démarche de Commons, nous considérons ici que les origines légales (Glaeser et Shleifer, 2002) sont loin d'épuiser l'explication d'une diversité des systèmes juridiques. Celle-ci gagne à être abordée à partir de l'hypothèse de complémentarité des normes (légales et sociales) comme élément explicatif d'un modèle de gouvernance d'entreprise ou d'un mode de production. Nous voulons ici montrer qu'un groupe d'innovations institutionnelles, parmi lesquelles figure la contrainte par corps, a contribué à l'émergence et au développement d'un capitalisme financier. Droit des sociétés et droit des faillites constituent ainsi les deux facettes d'un même système de gouvernance modelé par des 
groupes d'intérêt, des contraintes structurelles et une idéologie (Skeel, 1998) et le passé. Les législateurs de l'époque ne peuvent pas avoir été indifférents à ces formes de complémentarité.

\subsection{L'avènement de la responsabilité limitée et la protection du débiteur}

Si les premières sociétés par actions sont nées au 13ème siècle ${ }^{26}$, le 19ème est celui des innovations en matière juridique qui permettent de limiter la responsabilité des commerçants en dissociant de plus en plus nettement l'entreprise de l'entrepreneur (Despax, 1957, p. 8). Cela passe par la création de nouvelles formes juridiques d'entreprises et par la réduction des barrières à la création de sociétés, anonymes notamment. Mais l'encouragement de l'économie suppose aussi que les patrimoines des familles soient protégés des créanciers ce que le législateur a autorisé en introduisant l'inaliénabilité d'une partie du patrimoine.

\subsubsection{La création et la montée en puissance des SARL}

La responsabilité limitée prend sa pleine puissance, en France comme dans la plupart des autres pays occidentaux d'ailleurs, au 19ème siècle. A partir de l'Ordonnance de 1673, deux formes sociales permettaient cependant de protéger les apporteurs de capitaux davantage que ne le garantissait la forme d'association la plus courante, la Société en nom collectif (Lévy-Bruhl, 1938). La Société en commandite simple permettait aux commanditaires de disposer d'une responsabilité limitée (jusqu'à ce que la loi de 1856 les oblige à constituer un conseil de surveillance dont les membres peuvent voir leur responsabilité engagée), mais celle des commandités restait évidemment totale. La Société en commandite par action permettait d'aller plus loin en matière de désengagement dans la mesure où les parts des associés simples apporteurs de capitaux pouvaient être vendues. L'existence d'un marché des actions de ces commandites particulièrement actif au 19ème siècle a d'ailleurs permis aux associés de ces entreprises de profiter de nombreux avantages de la SA sans avoir eu besoin d'obtenir

\footnotetext{
${ }^{26} \mathrm{Au}$ milieu du 13e siècle des citoyens de Toulouse forment une société par action, la Société du Bazacle. Chaque action, appelée uchau, valait $1 / 8 \mathrm{e}$ de moulin. Les 96 actions représentaient donc les 12 moulins du Bazacle. Les actions sont échangées sur un marché libre et les propriétaires ont droit à des distributions de dividendes, à la lecture annuelle des comptes, de choisir des mandataires sociaux qui administrent la société pendant un an, d'acheter d'autres moulins ou de gérer les employés, etc. C'est à partir de 1370 que la société est réellement devenue une société anonyme, au sens où les porteurs n'avaient plus des parts de moulins mais bien les parts de la société possédant les moulins.
} 
l'autorisation d'en créer une (Lamoreaux \& Rosenthal, 2005). La responsabilité limitée est consacrée par le Code de commerce de 1807 avec la création de la société anonyme mais celle-ci restera soumise à autorisation administrative jusqu'en $1867^{27}$; c'est donc une responsabilité limitée sous surveillance qui a été mise en place par le législateur. Son champ d'application est réduit également: seules 642 SA ont été créées entre 1800 et 1867, (Freedeman, 1979 et 1993)

Les besoins de création de société sont cependant satisfaits par l'existence des commandites qui permettent de répondre à l'une des principales attentes d'un propriétaire-dirigeant, la consolidation de sa place dans l'entreprise. Posant la question dans les termes de la théorie de l'agence, on soulignera ici que les différentes formes de commandites bloquent tout à la fois le risque de dilution du capital et celui d'abus de pouvoir de la part des principaux apporteurs de capitaux (Guinnane et al., 2007). Une fois passée la crise de 1857 les contraintes qui pèsent sur les sociétés anonymes et les abus commis par les participants à des commandites vont pousser à l'adoption d'une nouvelle forme sociale. Il s'agit de la Société à responsabilité limitée, créée par la loi du 23 mai 1863, dont le montant du capital est également limité 28 . En 4 ans, 52 sont créées pour 80 millions de capital. Peu à peu les contraintes dans ce domaine vont être relâchées au point que la SARL va rapidement devenir la forme sociale la plus utilisée.

\footnotetext{
${ }^{27}$ La loi du 23 mai 1863 autorise les entreprises dont le capital ne dépasse pas les 20 millions de francs de se constituer en sociétés anonymes sans avoir besoin de demander l'autorisation de l'état. Les contraintes qui pesaient sur les directeurs et les managers par la loi de 1857 continuaient cependant de s'appliquer et furent même accentuées par l'obligation faite aux administrateurs de posséder un vingtième du capital social sur lequel ils sont solidaires. La loi du 24 juillet1867, celle-là même qui abolit la contrainte par corps, a supprimé cette limite et a soumis les SA et les commandites par actions aux mêmes (moindres) exigences.

${ }^{28} \mathrm{La}$ création de SARL française a été anticipée par l'autorisation de poursuivre leur exploitation en France donnée aux SARL étrangères. La loi du 30 mai 1857 concernait les SARL belges et le traité du 30 avril 1862 les SARL anglaises.
} 
Figure 1: Les nouvelles entreprises en fonction de la forme juridique (1852-1978)

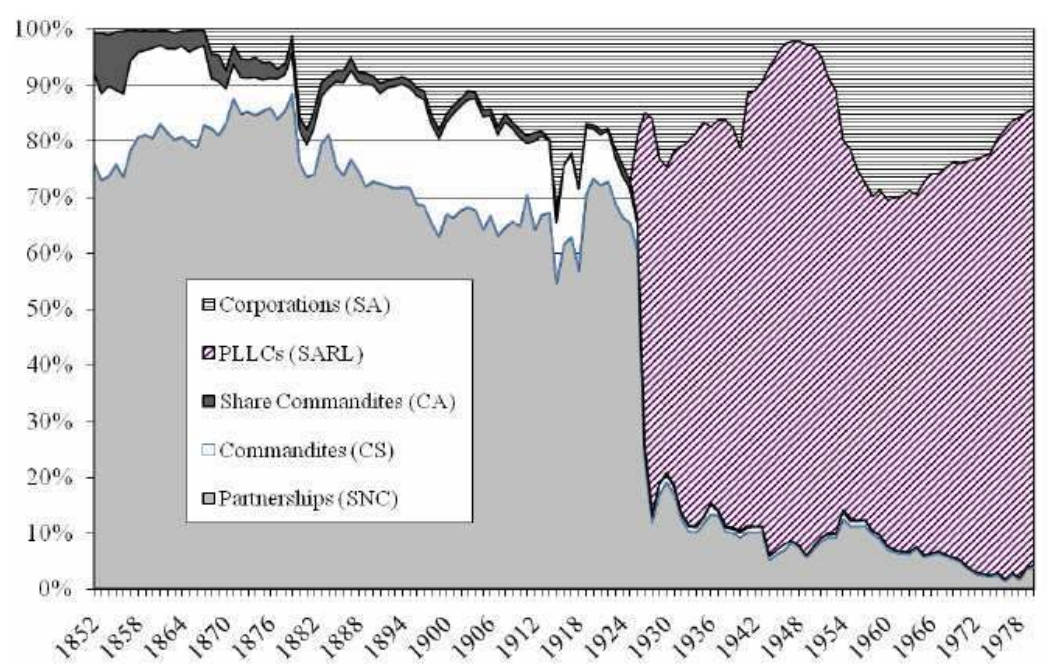

Source (Guinnane et al. 2007)

Le graphique ci-dessus retrace la répartition des nouvelles entreprises autres que les entreprises individuelles entre 1852 et 1978. L'effet de la loi de 1867 y apparaît dans une certaine mesure : on observe bien une augmentation de la part des SA. Celle-ci est cependant d'une ampleur assez limitée et de courte durée : les commandites simples regagnent du terrain dès la fin du 19ème siècle. Dans l'ensemble, les SA ne représenteront jamais plus de 10\% du total des créations de sociétés avant le début de la première guerre mondiale. Il est frappant d'observer le faible engouement suscité par la SARL, alors même qu'elle était réclamée par les acteurs économiques et qu'elle est réputée constituer la forme la mieux adaptée aux petites et moyennes entreprises. Au contraire, les Sociétés en commandite simple, qui engagent la responsabilité complète des associés impliqués dans la gestion de l'affaire, non seulement résistent bien à l'apparition de la nouvelle forme juridique mais connaissent même un certain succès. Comment expliquer la faible proportion des formes juridiques les plus protectrices après le vote de la loi du 24 juillet 1867 ?

Il ne semble pas que les coûts de constitution ou la fiscalité puissent être mis en avant pour expliquer cette désaffection. Si les impôts cédulaires, sur les bénéfices industriels et commerciaux en particulier sont créés par la loi du 31 juillet 1917, les impôts payés par les entreprises resteront indépendants de leur forme juridique 
jusqu'à la seconde guerre mondiale. Ce commentaire peut être étendu à toutes les contraintes administratives. A partir du moment où la loi de 1867 abolit les restrictions en matière de capital et les obligations des dirigeants mise en place en 1857, on ne peut pas considérer que les SA et les SARL constituaient des formes plus contraignantes que les commandites. Il est donc possible que le décollage limité des SA et SARL provienne de la bonne protection qu'offraient les commandites contre le risque de dilution du capital sans que celle-ci s'accompagne d'un danger accru de voir les actionnaires minoritaires chercher à imposer leurs préférences en matière de gestion aux dirigeants.

Même si elles ne sont pas directement adoptées par les protagonistes de la création d'entreprise, les nouvelles formes sociales créées par un droit des sociétés en expansion marquent l'avènement d'une rupture fondamentale introduite par la propriété mobilière. Elle est très tôt perçue par Marx: "D'une manière générale, les sociétés par actions -qui se développent avec le système du crédit- tendent de plus en plus à séparer cette fonction administrative d'avec la possession du capital [...] à côté du véritable manager apparaît une foule de conseils d'administration et de direction pour qui l'administration et la direction ne sont, en fait, que prétextes à spolier les actionnaires et à amasser les richesses" (Marx, 1968, pp.1148-1150.). On retrouve cette thèse d'un capitalisme modelé par les formes juridiques chez un auteur comme Ripert pour qui la dématérialisation croissante des patrimoines et la négociabilité des titres favorisent les comportements opportunistes et la prise de risque par les entreprises (Ripert, 1951, p. 156 et ss).

Toutefois, et c'est ce qui expliquera aussi les grandes ruines consécutives aux faillites de la fin du 19ème siècle, en même temps qu'elle protège les intérêts personnels et familiaux des entrepreneurs, la responsabilité limitée va leur permettre d'échapper aux risques qu'ils créent. Cette possibilité est dénoncée par Marx pour qui la question de la délinquance managériale, forme extrême de la possibilité d'assumer une petite partie du risque effectivement encouru, est consubstantielle au système : " [la société anonyme] fait renaitre une nouvelle aristocratie financière, un nouveau ramassis de parasites, en la personne de promoteurs d'entreprises et de directeurs (managers qui ne le sont que par le nom) ; tout un système de tripotages et d'escroqueries fondé sur le trafic d'actions, etc. C'est un mode de production privée qui échappe au contrôle de la propriété privée" (Marx, 1968, pp.1176-1177). Ces arguments relatifs à une finance prédatrice seront repris et développés par les tenants de la notion de capital financier et tout particulièrement Veblen (1904) et Hilferding (1910) Pour ces auteurs, l'anonymat aurait en effet pour conséquence de dissoudre la responsabilité personnelle et favoriser les dérapages, en particulier spéculatifs. 
Cette possibilité d'échapper à la responsabilité n'est donc pas le résultat d'une dérive comportementale des actionnaires et dirigeants. Elle s'inscrit au contraire dans les statuts mêmes de l'entreprise (Collin, 1996). Les efforts de cadrage entrepris à travers les réformes successives du droit des faillites notamment, pour réguler et sécuriser le comportement d'entreprises dont le patrimoine est de plus en plus immatériel vont être complétés par des dispositions relatives à la nature des gages qui peuvent être saisis et à l'augmentation du périmètre des biens de l'emprunteur qui peuvent être transférés au prêteur. Les modifications qui affectent le régime d'inaliénabilité du patrimoine relèvent de cette préoccupation.

\subsubsection{L'inaliénabilité du patrimoine}

A titre historique, deux tendances peuvent être identifiées en ce qui concerne le patrimoine et son transfert du débiteur au créancier en cas de non respect des engagements pris dans le cadre d'un contrat de prêt. D'un côté on trouve la protection des biens du débiteur et, de l'autre, les traces de la volonté des prêteurs d'aller aussi loin que possible dans la saisie des biens en cas de défaillance. Les deux orientations s'articulent sur le principe du droit des créanciers défini par les articles 2284 et 2285 du Code civil qui définissent le droit de gage général des créanciers en ces termes: "quiconque s'est obligé personnellement, est tenu de remplir son engagement sur tous ses biens mobiliers et immobiliers, présents et à venir " et "les biens du débiteur sont le gage commun de ses créanciers (...) ". Le droit de gage général met en relief le fait qu'il existe un lien juridique entre les dettes d'une personne et l'ensemble de ses biens et de ses droits évaluables en argent. En cela, il façonne la relation de dette.

Le régime dotal, s'est longtemps caractérisé par l'inaliénabilité des biens dotaux, ainsi préservés de la mauvaise gestion du mari (Code civil, art. 1554 et s., abrogés par la loi du 13 juillet 1965). L'inaliénabilité est également utilisée dans l'institution des biens de famille, apparue en 1909 pour endiguer l'exode rural au profit des petites propriétés (Code de la famille, art. 32 et art. 10 de la loi du 12 juillet 1909). Pourtant, la possibilité d'exclure une partie des biens contenue dans la clause d'inaliénabilité va rapidement devenir source de conflits entre le créancier du gratifié et le disposant. D'un côté, le créancier peut légitimement s'attendre à pouvoir s'emparer du bien, dans la mesure où il fait partie du patrimoine du débiteur, dont l'article 2092 du Code civil précise qu'il constitue l'assiette du droit de gage général du créancier. Il est donc fondé à réclamer la garantie attachée à l'engagement personnel de son débiteur. A l'inverse l'argument le plus souvent avancé en faveur du disposant est qu'il a transmis gratuitement le bien au débiteur, de sorte qu'il 
pouvait parfaitement restreindre la propriété du gratifié afin d'immobiliser le bien à son service. Partant de là, il n'a jamais entendu rendre le bien accessible aux créanciers du gratifié, qui n'ont d'ailleurs pas à se plaindre puisque le bien ne ferait pas partie du patrimoine de leur débiteur sans la générosité du disposant. Sa volonté profitable au débiteur et ses créanciers doit donc être respectée au mieux. Cet antagonisme s'explique évidemment par les finalités différentes qu'ont le droit de la famille et le droit des créanciers. Le droit de la famille favorise les intérêts familiaux, or la clause d'inaliénabilité est conçue essentiellement dans ce but. Le droit des créanciers, à cheval sur le droit des obligations et les voies d'exécution, vise au désintéressement des créanciers, donc est moins sensible aux considérations plus morales que patrimoniales qui justifient la clause d'inaliénabilité.

Longtemps la règle de l'insaisissabilité conséquence inévitable de l'inaliénabilité n'était guère remise en cause. On considérait que ce postulat était imposé par la logique, suivant laquelle l'inaliénabilité ne pouvait être efficace qu'en ayant pour corollaire l'insaisissabilité. Cette idée s'imposait d'autant plus qu'on retrouve la même règle dans l'inaliénabilité des biens de famille, ou encore à titre historique inaliénabilité des biens de la femme dans l'ancien régime dotal. Au demeurant l'analyse la plus souvent faite de la clause d'inaliénabilité est qu'elle frappe le bien d'indisponibilité réelle, donc est opposable à tous, notamment aux créanciers. L'idée est que le bien est immobilisé, affecté au service d'un intérêt particulier. Le titulaire de cet intérêt aurait en quelque sorte un droit réel sur le bien, limité à la satisfaction de son intérêt.

Ce type de disposition n'avantage pas l'engagement des prêteurs dans les projets des entreprises. C'est pourquoi on trouve la trace de nombreux rapports et lettres demandant la restriction, voire la suppression, du principe d'inaliénabilité qui serait à l'origine d'une moindre confiance de la part des créanciers. L'extension du domaine des biens qu'ils peuvent saisir en cas de défaillance de l'emprunteur fait d'ailleurs partie des demandes permanentes de ce groupe d'acteurs, notamment depuis que la dette bancaire est devenue le principal mode de financement de l'activité économique. La possibilité de couvrir les dettes par le transfert de droits de propriété est cependant rapidement devenue insuffisante à satisfaire les besoins de financement nés de la croissance de l'activité économique et de l'accumulation au cours de la seconde moitié du 19ème siècle. D'où l'apparition d'un nouveau type de gage assis non plus sur des actifs préalablement acquis et/ou accumulés mais sur des anticipations de gains futurs. 


\subsection{Du gage à la promesse : l'expression de la futurité}

La transition d'une société agrarienne vers une société commerciale s'accompagne aussi d'interrogations sur l'ancrage de valeurs qui sont avant tout des anticipations sur l'avenir. Dans ce domaine aussi, les innovations caractérisent le 19ème siècle (voir Baubeau, 2007, qui présente le cas du warrant agricole et comment, à la différence du warrant commercial il a permis de ne pas déplacer le gage). Nombre d'entre elles font écho au besoin accru de financement et correspondent à ce que David Hume avait anticipé en qualifiant l'économie naissante de "société de crédit " (Cf. Desmedt, 2003). A la différence de l'économie essentiellement agraire dans laquelle l'instabilité et l'incertitude paraissent relativement circonscrites, l'économie de crédit se caractérise par une expansion des engagements financiers et une prolifération de promesses. A leur propos, Hume évoque une certaine formule verbale " par laquelle nous nous engageons à accomplir une action. Cette formule verbale constitue ce que nous appelons une promesse, sanction du commerce intéressé entre les hommes." (Hume, 1739-1740, p.641). Avec la promesse, des liens se forgent entre l'énonciateur et les récepteurs d'une parole, donc entre l'endetté et ses créanciers. Dans ce système, la promesse de remboursement matérialisée par la signature d'un titre financier constitue un engagement crucial. Pour éviter que le marché soit purement rhétorique, afin de donner une forme concrète aux promesses et de manière à contrôler les passions déstabilisatrices, il convient de déployer certains modèles d'action générateurs de stabilité. C'est le rôle de l'état à qui il revient de mettre en place un système de gouvernement permettant de discipliner les formes passionnelles ou, dit autrement, de faire respecter les contrats.

Il est à ce propos frappant de remarquer la simultanéité presque parfaite entre l'abolition de la contrainte par corps et l'avènement de la responsabilité limitée. Gustave de Molinari (1863) le souligne déjà dans la troisième partie intitulée "De la circulation " de son Cours d'Economie Politique. Après avoir rappelé les deux manières d'engager des capitaux dans la production, l'engagement direct dans une affaire ou le prêt, et avoir identifié les formes de rémunération (profit/dividende ou intérêt) ainsi que leurs risques respectifs, il précise les caractéristiques de la relation de prêt et le besoin de garanties qu'elle appelle : "En quoi peut consister cette sécurité? D'abord, dans les gages que m'offre l'emprunteur-assureur. Ces gages peuvent affecter autant de formes qu'il existe de modes d'investissement des valeurs. L'emprunteur peut m'offrir en gage des valeurs personnelles, immobilières et mobilières". Retraçant l'histoire du prêt et de ses différents supports, Molinari met alors en évidence la dématérialisation opérée au fil du temps. Il montre 
d'abord comment l'association du prêt à la personne est caractéristique des systèmes économiques anciens et prend soin de préciser que la contrainte par corps est un reste de ce régime. Les valeurs immobilières s'y sont substituées dans les prêts avec hypothèque. Mais la prise d'hypothèque sur les valeurs mobilières a bouleversé ce modèle de prêt. L'incertitude et le risque qui s'y attachent ne peuvent en faire des garanties satisfaisantes pour les prêteurs, d'où la nécessité d'autoriser légalement d'autres formes contractuelles.

C'est le cas par exemple de l'autorisation de nantissement du fonds de commerce qui va occuper une bonne partie des tribunaux et des débats législatifs au 19ème siècle. La volonté de l'inclure parmi les éléments de l'actif est assez ancienne. Stanziani (2007) note que, dès la seconde moitié du 18ème siècle, les actes notariés et les bilans des faillites des marchands parisiens incluent la valeur du fonds compréhensif non seulement des murs et des stocks, mais également de l'achalandage. Cette solution suscite les réactions négatives des créanciers et des tribunaux consulaires qui estiment que l'achalandage sert à gonfler l'actif et que sa présence au bilan est superflue car il reste insaisissable par les créanciers. Les rédacteurs du Code de commerce resteront captifs des catégories de l'ancien régime et, alors même qu'il y est maintes fois fait référence au cours des travaux préparatoires, la notion de fonds de commerce demeure absente aussi bien du Code civil que du Code de commerce. Sur des bases aussi défavorables, comment un droit commercial aussi "backward looking" est-il parvenu à intégrer un élément intangible comme le fonds de commerce dont la principale caractéristique est d'être une condition nécessaire à la réalisation de recettes à venir ?

Il semble que la mobilité géographique à l'origine des ventes de plus en plus fréquentes de fonds de commerce ait incité l'administration fiscale de l'époque à innover dans ce domaine. Ainsi, afin de limiter les fraudes, un droit sur la cession de clientèle avait-il été introduit dès 1798, supposant ainsi la reconnaissance de l'achalandage d'un point de vue fiscal. Les distorsions de taux qui frappent les différentes assiettes taxées (marchandises neuves et clientèle) favorisent cependant les fraudes et dissimulations. Le lobby des commerçants va cependant obtenir gain de cause en 1898 avec l'autorisation $\mathrm{du}$ nantissement $\mathrm{du}$ fonds de commerce. Cette opération transforme le commerçant en capitaliste (Ripert, op. cit.) en même temps qu'elle autorise à emprunter sur la base non pas d'une garantie immobilière, foncière en particulier, mais sur celle des potentialités de l'activité concernée. En cela elle soutient le passage du capitalisme foncier au capitalisme financier.

La possibilité de gager des biens futurs offre des perspectives de financement plus larges que celles qui résultent du seul nantissement, opération qui revient à rendre liquide un actif 
antérieurement immobile. Par la même occasion, elle étend le champ de la richesse en la dégageant de l'accumulation de biens existants (la richesse usuelle au sens de Commons, 1934, p. 74) pour autoriser la prise en compte des droits de propriété sur des actifs à venir. La distinction entre "assets" et "wealth" opérée par Commons (pour une présentation des trois formes de propriété chez Commons, voir Theret, 2001) étaie, en même temps qu'elle recoupe, les différentes formes de capitalisme. Dans la période féodale et agricole, la propriété était principalement corporelle. Dans la période mercantile (17ème siècle en Angleterre), la propriété devint la propriété incorporelle des dettes négociables. Dans le capitalisme des dernières quarante années, la propriété devient aussi la propriété intangible (...) " (Commons, 1934, p. 76), propriété intangible qui “ est l'ensemble des attentes de transactions profitables futures (...)" (ibid.), " la valeur présente du futur pouvoir de marchandage des capitalistes" (ibid., p. 651). L'idée de futurité est essentielle à cette généralisation de l'espace des transactions. Elle introduit en effet la dimension du temps (délai) entre la transaction légale et la production-consommation du produit, étant entendu que la valeur découle du contrôle légal et pas du contrôle physique, "... les deux transferts de contrôle ne se confondant que lorsque le futur le moment qui sépare le contrôle physique du transfert de contrôle légal) est ramené à zéro " (Theret, 2001, p. 12).

En offrant la possibilité de s'engager dans une relation de prêt sur la base d'actifs soit intangibles, soit inscrits dans le futur sous la forme d'une promesse, le législateur du 19ème prolonge une tradition qui, au préalable, était cantonnée à des cercles d'acteurs ou à des opérations particulières. La systématisation de la valeur de l'engagement sur le futur traduit une nouvelle forme d'articulation entre sphère politique et gestion financière qui cherche à étendre la possibilité de financement dont peuvent disposer des agents économiques, personnes physiques ou morales, impliquées dans le processus d'accumulation du capital. La professionnalisation des prêteurs, la montée en puissance de la dette financière à côté de la dette commerciale, la croissance de la taille des entreprises et la persistance d'un monde de petits boutiquiers appellent en effet des innovations en matière financière grâce auxquelles l'offre de crédit pourra s'adapter au développement des transactions réelles. Le développement de la promesse comme base du contrat de crédit, la responsabilité limitée et la protection du débiteur et de sa famille n'auraient pu émerger dans un système juridique prévoyant l'emprisonnement du débiteur pour dettes. En abolissant ce dernier dispositif, déjà tombé en désuétude dans les tribunaux mais toujours présent dans le "droit des livres", le législateur a autorisé le développement de nouvelles pratiques de financement et marqué la 
généralisation d'un capitalisme financier jusqu'alors présent en germe dans les interstices du capitalisme patrimonial.

\section{Conclusion}

L'abolition de la contrainte par corps pour dette commerciale au 19ème siècle fait partie des transformations du droit qui accompagnent les mutations de l'économie et qui, à la même époque, vont donner naissance à une généralisation des logiques marchandes issues de la constitution d'un système de prix lié à l'organisation même de la production.

Cette évolution est particulièrement évidente en matière de crédit. Depuis le XIIe siècle au moins, ce dernier s'appuyait sur deux éléments principaux : d'une part, les garanties formelles, la propriété immobilière notamment, et, d'autre part, la réputation et le capital immatériel de l'emprunteur. Capacité de remboursement héritée du passé et perspectives de gains dans le futur déterminent alors simultanément les conditions de la relation entre créanciers et débiteurs. Ces aspects jouent certes différemment suivant les situations particulières, les acteurs et la conjoncture. Leur prise en compte conjointe par les créanciers se présente néanmoins comme une constante de la relation d'endettement. Elle s'appuie sur une assise financière préalable du débiteur pour des raisons de sécurité, mais devient un pari sur des attentes de recettes futures pour des motifs de profit. Cette double perspective est source de tensions entre garanties formelles immobilières et capital intangible. Elle intervient également dans la caractérisation des différentes phases historiques du capitalisme. Le XIXème siècle la voit s'exprimer avec le plus d'intensité dans divers pays d'Europe malgré certaines différences de temporalité. L'explosion de multiples formes de crédit (commercial, personnel, bancaire), des motifs d'endettement (investissement, exploitation, consommation) et des échéances ainsi que les crises qui accompagnent ce mouvement illustrent les difficultés des prêteurs à équilibrer les garanties passées et les revenus futurs.

La suppression de la contrainte par corps pour dette commerciale du code de commerce français s'inscrit enfin dans une forme de système de "juge entraineur " (Ost, 1983). Dans un premier temps, son faible usage par les tribunaux permet aux débiteurs de s'affranchir des obligations habituellement associées à la dette et à dissocier le remboursement de la possession de garanties tangibles. La systématisation de l'abandon de cette pratique qui disparait du droit des livres en 1867 ouvrira plus largement une voie de transformation des pratiques financières dans laquelle l'économie est déjà engagée. En cela, cette innovation autorise la survenance d'une transformation au caractère systémique indiscuté et marque le 
passage d'un capitalisme patrimonial à un capitalisme financier. Ces catégories sont des idéal-types au sens de Weber. Elles permettent également d'avoir un système d'explication complet qui incorpore l'économique, le social et le juridique et peut s'étendre jusqu'à la manière que l'économie politique les appréhende. Car les innovations dans le domaine financier et en matière de statut juridique des entreprises qui se produisent à l'époque ne resteront pas non plus sans effet sur le contenu de l'économie politique qui de science de la liberté individuelle qu'elle était au 18ème siècle va se transformer en une "...science de la création, de la négociabilité, de l'extinction et de la rareté de la dette" (Commons, 1934, p. 934)

\section{Références}

ALAUZET, Isidore (1857) Commentaire du Code de Commerce et de la Législation Commerciale, Tome 4ème, Paris, Cosse et Marchal.

ANONYME (1860) La Contrainte par corps au 19ème siècle, Paris, Typographie Alexandre Lebon.

ASSELAIN, Jean-Charles (1984) Histoire Economique de la France, Volume 1, Paris, Editions du Seuil.

ATIYAH, Patrick S. (1979) The Rise and Fall of Freedom of Contracts, Oxford, Clarendon Press.

BALZAC, Honoré de (1837) Illusions perdues, Paris, Jamar. Réédité par la Librairie Générale Française, Paris, 1962.

BAUBEAU, Patrice (2007) The warrant agricole (1880-1914) : interstitial form or makeshift legal innovation ?, Communication au colloque "Not just firms: History, Law and Economics", PSE, Paris, mars.

BAYLE-MOUILLARD, Jean-Baptiste (1836) De l'emprisonnement pour dettes, considérations sur son origine, ses rapports avec la morale publique et les intérêts du commerce, des familles, de la société suivies de la statistique de la contrainte par corps, Paris, Imprimerie Royale.

BRAVARD-VEYRIERES, P. (1840) Manuel de Droit Commercial, $2^{\text {ème }}$ édition, Paris, Joubert.

BRISSAUD, Jean (1908) Manuel d'Histoire du Droit Privé, Paris, Fontemoing.

CHAPUT, Yves (1996) Droit des entreprises en difficulté et faillite personnelle, Paris, Presses Universitaires de France.

CLAUSTRES, Julie, (2007) La dette, la haine et la force : les débuts de la prison pour dette à la fin du Moyen Âge, Revue Historique, $\mathrm{n}^{\circ}$ 644, pp. 797-821.

COLLIN Sven-Olof (1996) Bad Losers: An Investigation of the Morality of the Limited Liability of Shareholders in a Joint Stock Company; Journal of Economic Issues, Vol. 30, ${ }^{\circ}{ }^{\circ}$, pp. 283-289. 
COMMONS, John R. (1934) Institutional Economics. Its Place in Political Economy, Madison, The University of Wisconsin Press (1961 reprint).

DALLOZ, Ainé et Armand DALLOZ (1851) Répertoire de Législation, de Doctrine et de Jurisprudence, Tome 12, Paris, Bureau de la Jurisprudence Générale.

DALLOZ Edouard et C. VERGE (1877) Les Codes Annotés. Code de Commerce annoté et expliqué d'après la jurisprudence et la doctrine, Bureau de la Jurisprudence générale, Paris.

DEFFAINS, Bruno (2007) Introduction à l'analyse économique des systèmes juridiques, Revue Economique, 58-6, pp. 1149-1162.

DESMEDT, Ludovic (2003) Finance et Entreprises : entre Logiques Disciplinaires et Dynamiques Déstabilisatrices, Communication au Forum de la Régulation.

DESPAX, Michel (1957) L'Entreprise et le Droit, Paris, LGDJ.

DESURVIRE, Daniel (1991) Banqueroute de l'antiquité à la France contemporaine, Les Petites Affiches, 30 août, p. 12 et 2 septembre, p. 4.

DI MARTINO, Paolo (2005) Approaching disaster, a comparison between personal bankruptcy legislation in Italy and England, 18801930, Business History, XLVII, 1, pp. 23-43.

FARRAR, John (1981) The Rise and Fall of Freedom of Contract: Book review, The British Journal of Law and Society, winter, ${ }^{\circ} 2$, pp. 277-280.

FERRIERE, Claude-Joseph, de (1755) Dictionnaire de droit et de pratique, Paris, nouvelle édition.

FREEDEMAN Charles E. (1979) Joint-Stock Enterprise in France, 1807-1867: From Privileged Company to Modern Corporation, Chapel Hill: University of North Carolina Press.

FREEDEMAN Charles E. (1993) The Triumph of Corporate Capitalism in France, 1867-1914, Rochester, New York, University of Rochester Press.

GILLE, Bertrand (1959) La banque et le crédit en France de 1815 à 1848, Paris, Presses universitaires de France, Mémoires et documents publiés par la Société de l'Ecole des chartes.

GLAESER, Edward et Andrei SHLEIFER (2002) Legal Origins, Quarterly Journal of Economics, ${ }^{\circ} 117$, pp. 1193-1230.

GUINNANE, Timothy W., Ron HARRIS, Naomi R. LAMOREAUX, et Jean-Laurent ROSENTHAL (2007) Ownership and Control in the Entrepreneurial Firm: An International History of Private Limited Companies, décembre, Yale University Economic Growth Center Discussion Paper No. 959 (Disponible sur SSRN: http://ssrn.com/abstract $=1071007$ )

GUYOT, Yves et Arthur RAFFALOVITCH (1901) Dictionnaire du commerce, de l'industrie et de la banque, Paris, Guillaumin et Cie. 
HAUTCOEUR, Pierre-Cyrille (2008) La statistique et la lutte contre la contrainte par corps. L'apport de Jean-Baptiste Bayle-Mouillard, Histoire et Mesure, XXIII-1, pp 85-136.

HAUTCOEUR, Pierre-Cyrille et Nadine LEVRATTO (2007a) Faillite, in STANZIANI, A. (sous la direction de) Dictionnaire de l'Economie-Droit (XVIIIème-XXème siècle), Paris, LGDJ, pp. 159-168.

HILAIRE, Jean (1992) Introduction Historique au Droit Commercial, Paris, PUF.

HILFERDING, Rudolf (1970) Le Capital Financier, Paris, Les Editions de Minuit. Première édition originale, 1910.

HOFFMAN, Philip T., POSTEL-VINAY, Gilles \& ROSENTHAL, JeanLaurent (2001) Des marchés sans prix. Une économie politique du crédit à Paris, 1660-1870, Paris, Éditions de l'EHESS.

HUME, David (1739-1740) Traité de la Nature Humaine, Aubier Paris, Montaigne, 1983.

KALNIN-MAGGIORI, Hélène (2004) Libertas dans la Première Décade de l'Histoire Romaine de Tite-Live, Thèse de doctorat de l'université de Lille 3, discipline : Latin.

LAINNE, M.A.F. (1839) Commentaire analytique de la loi du 8 juin 1838 sur les faillites et banqueroutes contenant le texte de la loi, l'examen des dispositions nouvelles qu'elle renferme et le résumé complet de la doctrine et de la jurisprudence sur les dispositions, Corbeil, Imprimerie de Crété, VI-676 p.

LAMOREAUX Naomi R. et Jean-Laurent ROSENTHAL (2005) Legal Regime and Contractual Flexibility: A Comparison of Business's Organizational Choices in France and the United States during the Era of Industrialization, American Law and Economics Review, $\mathrm{n}^{\circ} 7$, pp. 28-61.

LESCURE, Michel et Alain PLESSIS [dir.] (1999) Banques locales et banques régionales en France au XIXe siècle, Paris, Éditions Albin Michel.

LESTER, V. Markham (1995) Victorian Insolvency Bankruptcy, Imprisonment for Debt, and Company Winding-up in NineteenthCentury England, Oxford University Press.

LEVRATTO, Nadine et Alessandro STANZIANI (2009) Introduction à l'ouvrage Le Capitalisme au Futur Antérieur - Crédit et spéculation en France, XIXe-XXe siècles, à paraître, Bruxelles, Bruylant.

LEVY-BRUHL, Henri (1938) Histoire juridique des sociétés de commerce en France aux XVIIe et XVIIIe siècles. Paris, DomatMontchrestien.

LUCAS, Charles (1836) De la Réforme des Prisons ou de la Théorie de l'Emprisonnement, de ses Principes, de ses Moyens et de ses Conditions Pratiques, Tome Premier, Paris, Editions Legrand et Bergounioux.

MARCO, LUC (1992) La Montée des Faillites en France, 19e-20e siècles, Paris, L'harmattan. 
MARTIN, Xavier (2003) Mythologie du Code Napoléon. Aux soubassements de la France moderne, Bouère, Éditions Dominique Martin Morin.

MASCRET, Henry-François (1863) Dictionnaire des faillites, [18481913], Chez l'auteur, Paris.

MARX, Karl (1968) Le Capital, Livre 3ème, 1864-1875, in OEuvres, Economie, T.II, Paris, Gallimard.

MERLIN DE DOUAI, PHILIPPE-ANTOINE (1812-1826) Répertoire universel et raisonné de Jurisprudence, $4^{\mathrm{e}}$ édition, Paris, Garnery.

MOLINARI, Gustave de (1863) Cours d'Economie Politique, Paris, Guillaumin (http://oll.libertyfund.org/title/653/104237 on 200712-13)

MOREAU-CHRISTOPHE, Louis (1841) Les pauvres : physiologie de la misère, texte établi sur un exemplaire (BM Lisieux : 4866) des Français Peints par Eux-Mêmes : Encyclopédie Morale du XIXe Siècle, Tome 4, publié par L. Curmer de 1840 à 1842 en 10 vol.

OST, François (1983) Juge-pacificateur, juge-arbitre, jugeentraîneur. Trois modèles de justice, in Gérard Philippe, Michel Van de Kerchove et François Ost (dir) Fonction de juger et pouvoir judiciaire : transformations et déplacements, Bruxelles, Facultés universitaires Saint-Louis, pp. 1-70.

POLANYI, Karl (1983) La Grande Transformation, Paris, Gallimard.

RIPERT, Georges (1951) Aspects Juridiques du Capitalisme Moderne, Paris, LGDJ (Réédition de 2004)

SCHWARTZ Alan (1998) A contract theory approach to business bankruptcy, The Yale Law Journal, Vol. 107, n6, pp. 1807-1851.

SGARD, Jérôme (2005) The liberalization of bankruptcy law in Europe, document de travail, CEPII.

SKEEL, David A. (1998) An evolutionary theory of corporate law and corporate bankruptcy, Vanderbilt Law Review, octobre, Vol. 51, $\mathrm{n}^{\circ} 5$, pp. 1326-1398.

STANZIANI, Alessandro (2007) : Fonds de Commerce, in STANZIANI, A. (sous la direction de) Dictionnaire de l'Economie-Droit (XVIIIèmeXXème siècle), Paris, LGDJ, pp. 185-194.

SZRAMKIEWICZ, Romuald (1989) Histoire du droit des affaires, Paris, Editions Montchrétien,

TEULET, Alexandre (1835) Dette, in Duckett, William (sous la direction de) Dictionnaire de la Conversation et de la Lecture, Tome XX, Paris, Belin-Mandar Librairie, pp. 360-363.

THERET, Bruno (2001) Saisir les faits économiques : une lecture structuraliste génétique de la méthode Commons; Cahiers d'Economie Politique, $\mathrm{n}^{\circ}$ 40-41, pp. 79-137.

TRIPIER, Louis (1902) Les Codes Français Collationnés sur les Editions Officielles, Paris, Librairie de Jurisprudence de Cotillon.

VAUSE, Erika (2007) In the Red and in the Black: Debt Imprisonment and the Culture of Credit in France, 1793-1867, Paper presented at 
the Workshop on Money, Markets and Consumption, Council on Advanced Studies in Humanities and Social Sciences, University of Chicago, Spring Quarter.

ZAMBRANA MORAL, Patricia (2004) Histoire de six articles du Code civil français : Les droits du débiteur, honneur et contrainte par corps, Revue Historique de Droit Français et Etranger, vol. $82, n^{\circ} 4$, pp. 589-611.

VEBLEN, Thornstein (1904) The Theory of Business Enterprise, Réédité par Transaction Publishers, 1978. 